\title{
Entangled Light from White Noise
}

\author{
M. B. Plenio ${ }^{1}$ and S. F. Huelga ${ }^{2}$ \\ ${ }^{1}$ Quantum Optics and Laser Science Group, Blackett Laboratory, Imperial College of Science, Technology and Medicine, \\ London, SW7 2BW, United Kingdom \\ ${ }^{2}$ Division of Physics and Astronomy, Department of Physical Sciences, University of Hertfordshire, Hatfield AL10 9AB, \\ United Kingdom
}

(Received 1 October 2001; revised manuscript received 12 February 2002; published 24 April 2002)

\begin{abstract}
An atom that couples to two distinct leaky optical cavities is driven by an external optical white noise field. We describe how entanglement between the light fields sustained by two optical cavities arises in such a situation. The entanglement is maximized for intermediate values of the cavity damping rates and the intensity of the white noise field, vanishing both for small and for large values of these parameters and thus exhibiting a stochastic-resonancelike behavior. This example illustrates the possibility of generating entanglement by exclusively incoherent means and sheds new light on the constructive role noise may play in certain tasks of interest for quantum information processing.
\end{abstract}

DOI: 10.1103/PhysRevLett.88.197901

PACS numbers: 03.65.Ud, 03.67.-a, 05.40.Ca

Quantum entanglement holds the key for qualitatively new forms of information processing [1]. This discovery has fueled an increasing interest in fully understanding how to create, manipulate, and exploit entanglement, a resource which has no classical analog. From a practical point of view, creating and exploiting entanglement has become tantamount to minimizing the impact of noise. In any real experimental scenario, the unavoidable interaction of the quantum processor with its surroundings results in a decoherence process. As a consequence, whatever initial entanglement may be present in the system of interest, it will subsequently degrade. The system may then end up in a mixed state whose amount of entanglement does not suffice to overcome the performance of classically correlated states. It is therefore of paramount importance for the practical realization of quantum information processing protocols to engineer mechanisms to prevent or minimize the impact of environmental noise.

The general approach of research so far has been to try to isolate potential quantum information processors as much as possible from the environment. The existing approaches can be classified into three categories. Quantum error correction [2] uses redundant coding to protect quantum states against noisy environments. This procedure is successful provided the error rate is sufficiently small. It requires a significant overhead in resources, in the number of both additional qubits and additional quantum gates, which makes this technique challenging within the limitations of presently available technology. A more economical approach consists of exploiting the existence of so-called decoherence-free subspaces that are completely insensitive to specific types of noise, for example, dephasing [3]. This approach tends to require fewer overheads, but is only applicable in specific situations. As an example, Kielpinski et al. [4] recently showed how the lifetime of an entangled pair of ions held in an ion trap can be increased by an order of magnitude. A third theoretical approach consists of implementing loop control strategies, where the use of ancillary systems is avoided at the price of interacting with the quantum processor, using either deterministic [5] or stochastic [6] dynamic control. The modus operandi of all these strategies relies on either trying to shield the system from the environmental noise or actively restoring the corrupted dynamics to the ideal one. In this Letter we will explore the possibility of adopting a different strategy. Instead of attempting to combat noise, we use noise to play a constructive role in quantum information processing. We will focus here on the problem of generating entanglement when only incoherent sources are available and demonstrate that, indeed, controllable entanglement can arise in this situation.

The idea that dissipation can assist the generation of entanglement was put forward recently [7]. In a system comprising two atoms held in an optical cavity, it was shown that the decay of the cavity field can be employed to assist the preparation of a pure maximally entangled state of the ions inside the cavity. Without cavity decay, the reduced state of the two-ion system is an inseparable mixture at all times. Including photon leakage leads to the undesired parts of the global wave function to decay and, therefore, such terms are eliminated for sufficiently large times. This idea was then further elaborated to show that cavity decay can be used to establish entanglement between different cavities [8].

Our approach here is more radical. We will employ white noise, not as an exclusively passive, dissipative element but as the actual driving force of the system, and the only one. Consider the situation depicted in Fig. 1. An atomic system is surrounded by two distinct optical cavities initially prepared in the vacuum state. The system is driven by an external thermal field whose intensity will be characterized in terms of an effective photon number $n_{T}$. Both cavities may leak photons at rates $\kappa_{a}$ and $\kappa_{b}$. The 


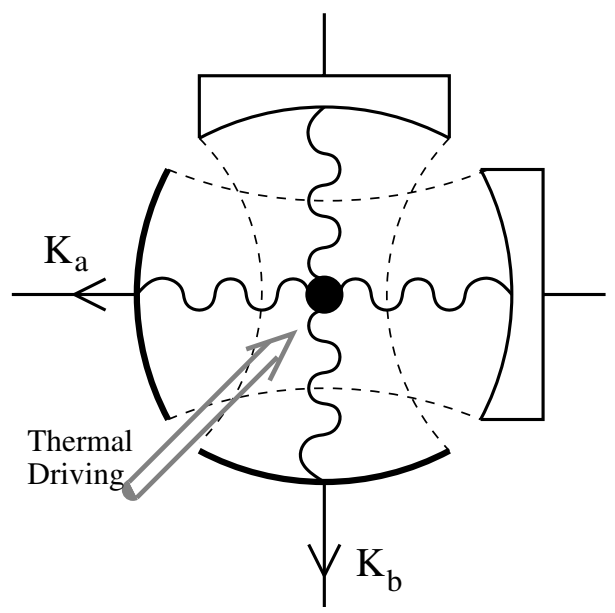

FIG. 1. Proposed experimental setup. An atomic system is surrounded by two optical cavities initially prepared in an arbitrary state and driven by a thermal field. Both cavities leak photons at rates $\kappa_{a}$ and $\kappa_{b}$. Despite the presence exclusively of incoherent processes the steady state solution of the system reveals that the cavity fields become entangled.
Bloch equations governing the time evolution of the global system are given by (in the following $\hbar=1$ )

$$
\dot{\rho}=-i[H, \rho]+\mathcal{L}(\rho),
$$

where the Hamiltonian $H$ describes the internal energies of the atom and the two cavities as well as the atom-cavity coupling. The Liouvillian $\mathcal{L}(\rho)$ describes the cavity decay and the interaction of the atom with the external thermal light field. As no external coherent driving is present, the Hamiltonian reads

$$
\begin{aligned}
H= & \nu_{a} a^{\dagger} a+\nu_{b} b^{\dagger} b+\omega|2\rangle\langle 2| \\
& +g_{a}\left(|2\rangle\langle 1|a+| 1\rangle\langle 2| a^{\dagger}\right) \\
& +g_{b}\left(|2\rangle\langle 1|b+| 1\rangle\langle 2| b^{\dagger}\right),
\end{aligned}
$$

where $g_{a(b)}$ is the coupling constant of the atom to cavity modes $a(b)$, and $|i\rangle$ denotes the internal state $i$ of the atom. The Liouvillian is given by

$$
\begin{aligned}
\mathcal{L}(\rho)= & -\kappa_{a}\left[a^{\dagger} a \rho+\rho a^{\dagger} a-2 a \rho a^{\dagger}\right]-\kappa_{b}\left[b^{\dagger} b \rho+\rho b^{\dagger} b-2 b \rho b^{\dagger}\right] \\
& -\left(n_{T}+1\right) \Gamma[|2\rangle\langle 2|\rho+\rho| 2\rangle\langle 2|-2| 1\rangle\langle 2|\rho| 2\rangle\langle 1|]-n_{T} \Gamma[|1\rangle\langle 1|\rho+\rho| 1\rangle\langle 1|-2| 2\rangle\langle 1|\rho| 1\rangle\langle 2|] .
\end{aligned}
$$

Here $\Gamma$ describes the coupling strength of the atom to the external fields and $n_{T} \Gamma$ is the transition rate due to the thermal field. The spectral width of the thermal field is large compared to the linewidth of the atomic transition so that its effect is that of a white noise source. Note that $n_{T}$ can be interpreted as an effective photon number and that spontaneous decay of the atom out of the cavities is included in this scenario via the $n_{T}+1$ term. To simplify the following considerations, we assume that $\nu_{A}=\nu_{B}=$ $\omega$ and chose an interaction picture with respect to $H_{0}=$ $\omega\left(|2\rangle\langle 2|+a^{\dagger} a+b^{\dagger} b\right)$. Using this transformation, the Liouvillian part remains unchanged, while the Hamiltonian part is now given by

$$
\begin{aligned}
H_{I}= & g_{a}\left(|2\rangle\langle 1|a+| 1\rangle\langle 2| a^{\dagger}\right) \\
& +g_{b}\left(|2\rangle\langle 1|b+| 1\rangle\langle 2| b^{\dagger}\right) .
\end{aligned}
$$

The general solution of Eq. (1) is extremely tedious. Therefore we study a special case that exhibits the typical behavior but which allows us to reduce the complexity, both analytical and numerical, of the problem considerably. This is the case where both cavities have the same decay rate, i.e., we assume $\kappa_{a}=\kappa_{b}$.

In this case we are able to introduce two new effective modes, one of which will be decoupled from the atom. This simple basis change is given by the definition of two new mode operators,

$$
c=\frac{g_{a} a+g_{b} b}{\sqrt{g_{a}^{2}+g_{b}^{2}}} \quad \text { and } \quad d=\frac{g_{b} a-g_{a} b}{\sqrt{g_{a}^{2}+g_{b}^{2}}},
$$

and corresponds to a beam-splitter transformation. In this new basis, the Hamiltonian and Liouvillian part of the master equation are given by

$$
H_{I}=g\left(c|2\rangle\left\langle 1\left|+c^{\dagger}\right| 1\right\rangle\langle 2|\right),
$$

where $g=\sqrt{g_{a}^{2}+g_{b}^{2}}$, and with $\kappa=\kappa_{a}=\kappa_{b}$,

$$
\begin{aligned}
\mathcal{L}(\rho)= & -\kappa\left[c^{\dagger} c \rho+\rho c^{\dagger} c-2 c \rho c^{\dagger}+d^{\dagger} d \rho+\rho d^{\dagger} d-2 d \rho d^{\dagger}\right] \\
& -\left(n_{T}+1\right) \Gamma[|2\rangle\langle 2|\rho+\rho| 2\rangle\langle 2|-2| 1\rangle\langle 2|\rho| 2\rangle\langle 1|]-n_{T} \Gamma[|1\rangle\langle 1|\rho+\rho| 1\rangle\langle 1|-2| 2\rangle\langle 1|\rho| 1\rangle\langle 2|] .
\end{aligned}
$$

It is straightforward to transform between the different mode pictures. For example, given a Fock state of the effective cavity modes $c$ and $d$, we can express it in terms of a linear combination of Fock states of the physical modes $a$ and $b$ via $\sqrt{m ! n !}|m\rangle_{c}|n\rangle_{d}=\left(c^{\dagger}\right)^{n}\left(d^{\dagger}\right)^{m} \mid$ vacuum $\rangle$. We observe that, due to the transformation to the new set of effective modes, we have one mode (mode $d$ ) which is completely decoupled from the Hamiltonian dynamics and is purely damped under the Liouvillian dynamics. As a consequence, irrespective of the initial state of mode $d$, it will not be populated in steady state. Therefore, we begin all our numerical investigations with both effective modes $c$ and $d$ in the vacuum state (which is equivalent to both physical modes $a$ and $b$ being in the ground state). Since the mode $d$ will then never be populated, we disregard mode $d$ in the following. With this approach both the analytical and the numerical integrations become much more efficient and we can now study the entanglement physics of our system. Nevertheless, the analytical expression for 
general parameter values is extremely tedious [9] and we begin with the presentation of some numerical results and then provide a physical explanation for them.

The typical behavior of the entanglement in the system is illustrated in Fig. 2. There we have plotted the amount of entanglement of the joint state of the two cavity modes (after tracing out the atomic system) as a two-variable function of the intensity of the thermal field (characterized by the effective photon number $n_{T}$ ) and time. The chosen parameters are $\nu_{a}=\nu_{b}=\omega, g_{a}=g_{b}=1, \kappa_{a}=\kappa_{b}=$ 1 , and $\Gamma=0.2$. This entanglement implies nonclassical correlations between the modes which are a resource in quantum communication tasks [1] or the violation of Bell inequalities [10]. It is quantified by the so-called logarithmic negativity $N(\rho)$, which is given by

$$
N(\rho)=\log \left\|\rho^{\Gamma_{B}}\right\|,
$$

where $\rho^{\Gamma_{B}}$ is the partial transpose of $\rho$ and $\left\|\rho^{\Gamma_{B}}\right\|$ denotes the trace norm of $\rho^{\Gamma_{B}}$, which is the sum of the singular values of $\rho^{\Gamma_{B}}$ [11]. We have chosen the logarithmic negativity as it has the advantage that it can be computed fairly easily for systems of arbitrary dimensions, in contrast to other established entanglement measures such as entanglement of formation or the relative entropy of entanglement [12]. $N(\rho)$ is non-negative and vanishes for states with positive partial transpose and, in particular, for separable states. In our simulation we cut off the intracavity photon number at a value of 3 (simulations with a cutoff of four photons lead to indistinguishable results). Note that, for any value of $t$, the behavior of the amount of entanglement between the cavity modes is nonmonotonic; it in-

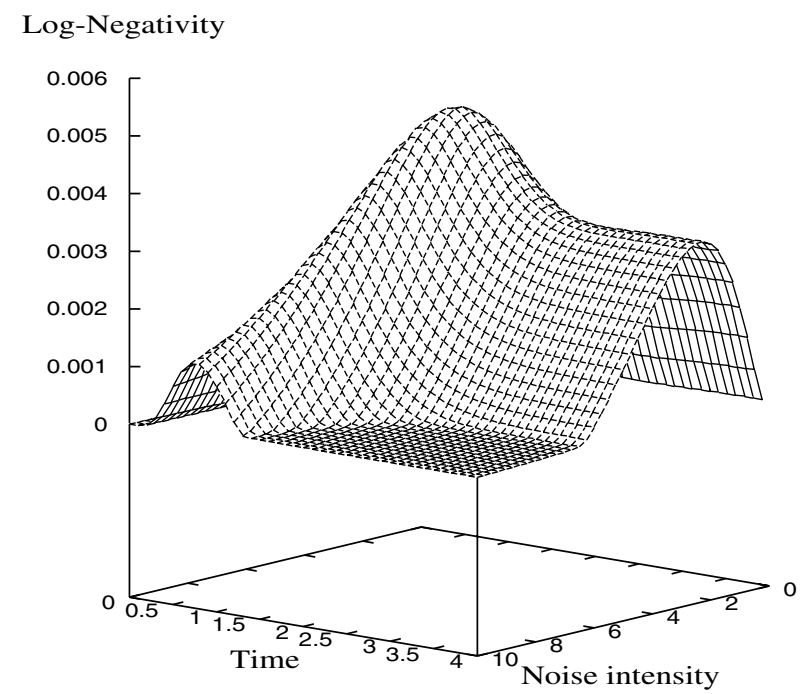

FIG. 2. Amount of entanglement $N(\rho)$, defined by Eq. (8), of the two-cavity field state as a function of the intensity of the external incoherent driving (characterized by the effective photon number $n_{T}$ ) and the time at which we determine the state of the light fields. We have chosen $\nu_{a}=\nu_{b}=\omega, g_{a}=$ $g_{b}=1, \kappa_{a}=\kappa_{b}=2$, and $\Gamma=0.2$. Note that the entanglement achieves its maximum for an intermediate intensity of the noisy driving field, a phenomenon reminiscent of stochastic resonance [13]. creases to a maximum value for an optimal intensity of the noisy driving field and then decreases towards zero for a sufficiently large intensity. This behavior is reminiscent of the well-known phenomenon of stochastic resonance [13] (see also [14]), where the coherent response of a system to a periodic signal is maximized in the presence of an intermediate amount of noise. This first approach where the atom is traced out may appear rather crude, and a more sophisticated approach would involve a measurement on the atom. Indeed, the expected amount of entanglement between the light fields can be increased if we subject the atomic system to a projective measurement in the $\{|1\rangle,|2\rangle\}$ basis. We have not plotted the resulting curves as they exhibit qualitatively the same behavior as in Fig. 2 but with an entanglement that is approximately twice as large.

It is also worthwhile to study the steady state entanglement of the cavity light fields as a function of the intensity of both the noisy driving field and the cavity decay rates. In Fig. 3 we present these result for $\nu_{a}=\nu_{b}=\omega$, $g_{a}=g_{b}=1, \kappa=\kappa_{a}=\kappa_{b}$, and $\Gamma=0.2$. The system exhibits a double resonance in both parameters, again reminding us of stochastic resonance. This demonstrates that both the noisy driving field and the cavity decay are necessary ingredients to generate steady state entanglement in this system. Note that a related effect assisted by cavity decay was found recently by Nha et al. [15] when studying the generation of squeezing in a coherently driven atom-cavity system. There, the squeezing is maximized as the cavity decay is increased to an optimal value and degrades for larger decay rates.

To understand the origin of the generation of entanglement from white noise and cavity decay let us begin by considering the special case of perfect cavities, i.e., $\kappa=0$. One easily checks that the stationary state of the joint

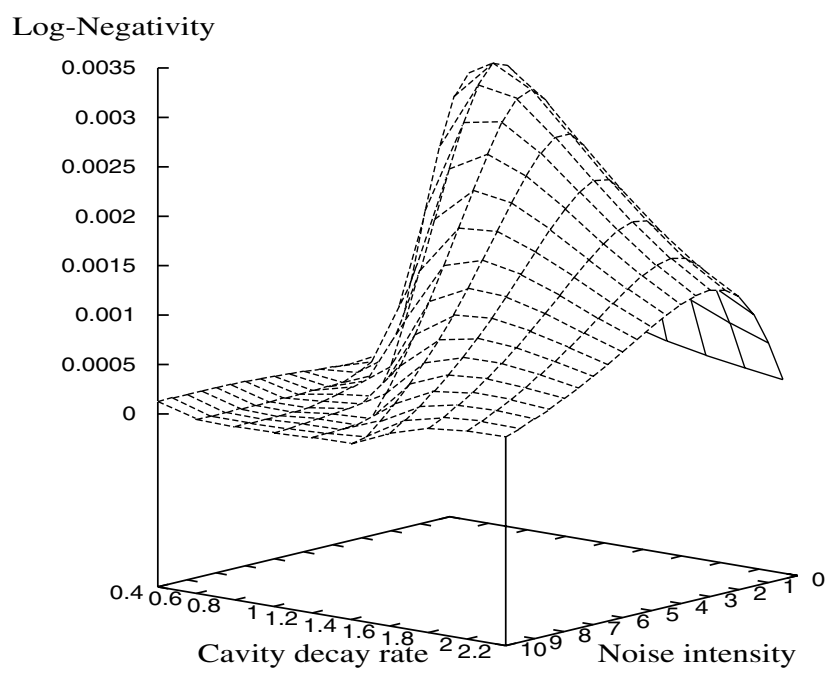

FIG. 3. The steady state entanglement of the two cavity modes as a function of both the cavity decay rate and the intensity of the noisy driving field. The parameters are $\nu_{a}=\nu_{b}=\omega, g_{a}=$ $g_{b}=1$, and $\kappa=\kappa_{a}=\kappa_{b}$. The system exhibits a resonance for both parameters. Entanglement persists only when both incoherent processes are present with moderate values. 
(atom-cavity field) system in the effective mode picture is then given by

$$
\rho^{s s}=\sum_{r=0}^{\infty} \sum_{i=1}^{2}\left(\frac{n_{T}}{1+n_{T}}\right)^{r+i-1} \frac{|i\rangle_{A}\langle i|\otimes| r\rangle_{C}\langle r|}{2 n_{T}+1},
$$

where $|r\rangle_{C}=\left(c^{\dagger}\right)^{r} \mid$ vacuum $\rangle / \sqrt{r !},|i\rangle_{A}$ denotes the internal atomic states, and $n_{T}$ is defined as in Eq. (3). This can also be written as

$$
\rho^{s s}=\sum_{r=0}^{\infty} \sum_{i=1}^{2}\left(\frac{n_{T}}{1+n_{T}}\right)^{r+i-1} \frac{|i\rangle_{A}\left\langle i|\otimes| \psi_{r}^{a b}\right\rangle\left\langle\psi_{r}^{a b}\right|}{2 n_{T}+1},
$$

where $\left|\psi_{r}^{a b}\right\rangle=\left(a^{\dagger}+b^{\dagger}\right)^{r} \mid$ vacuum $\rangle /\|\cdot\|$. Tracing out the atomic state results in a thermal distribution for the two cavity modes. Note that a thermal state of mode $c$ is also a mixture of coherent states and that the transformation back into the original two-mode picture (modes $a$ and $b$ ) corresponds to a beam-splitter transformation. A thermal state impinging on a beam splitter leads to a separable two-mode state after the beam splitter, and we conclude without further calculations that the state in Eq. (10) is not entangled.

If we increase the value of the cavity decay constant for fixed thermal noise intensity $n_{T}$, the stationary state is no longer a thermal mixture. For very large $\kappa$ only the lowest energy levels of the modes are occupied [the population of the effective mode $c$ with $r$ photons decreases as $(1 / \kappa)^{2+3(r-1)}$ for $\left.r \geq 1\right]$. A mixture of the vacuum and the one photon state in the effective mode picture transforms into a mixture of the vacuum and the triplet state $(|01\rangle+|10\rangle) / \sqrt{2}$. Such a mixture is always entangled as one can easily confirm by computing its partial transpose [12]. Therefore we expect that in the presence of cavity damping some entanglement will be present. This entanglement will again vanish in the $\kappa \rightarrow \infty$ limit as then the stationary state tends to the vacuum state.

To shed some more light on how exactly does the cavity decay generate the entanglement, consider the decay of a photon in the effective mode picture. Such a decay leads to the application of the operator $c$ to the state. In the original mode picture this corresponds to the application of the operator $a+b$. If this operator is applied to the state and the state is renormalized, then one observes that the relative weight of the entangled states in the mixture has increased. The conditional time evolution if there is no photon leakage has just the opposite effect, the mean number of photons decreases, i.e., the weight of entangled states in the mixture reduces [16].

To summarize, we have presented a preparation technique to entangle two light fields using only incoherent processes. Entanglement is present in the system at all times and its exact amount is a nonmonotonic function of the intensity of the external, incoherent driving. This be- havior resembles the phenomenon of stochastic resonance, where the response of a nonlinear system to weak periodic driving can be enhanced when supplemented with a noisy field of certain optimal intensity [13] (See [14] for proposed demonstrations in the quantum domain). In our case, the amount of entanglement of the two light fields is maximized for an optimal value of the external noisy field. If the intensity of the incoherent driving is increased beyond this optimal value, the negativity of the state decreases. This situation exemplifies the fact that tunable noisy sources can play a constructive role in certain situations and opens a new venue for exploring efficient ways to exploit the presence of noise when the aim is to generate entanglement in a controllable way.

We acknowledge comments from participants of the ESF-QIT conference in Gdansk, July 2001, where the main lines of this work were presented. This work was supported by EPSRC, the Nuffield Foundation, the EQUIP project of the European Union, the European Science Foundation Programme on "Quantum Information Theory and Quantum Computing," and the U.S. Army Research Office Grant No. 43371-PH-QC.

[1] M. A. Nielsen and I. L. Chuang, Quantum Computation and Quantum Information (Cambridge University Press, Cambridge, 2000); M. B. Plenio and V. Vedral, Contemp. Phys. 39, 431 (1998).

[2] P. W. Shor, Phys. Rev. A 52, 2493 (1995); A. R. Calderbank and P. W. Shor, Phys. Rev. A 54, 1098 (1996); A. Steane, Proc. R. Soc. London A 452, 2551 (1996).

[3] G. M. Palma et al., Proc. R. Soc. London A 452, 567 (1996); M. B. Plenio et al., Phys. Rev. A 55, 67 (1997); D. A. Lidar et al., Phys. Rev. Lett. 81, 2594 (1998).

[4] D. Kielpinski et al., Science 291, 1013 (2001).

[5] H. M. Wiseman and G. J. Milburn, Phys. Rev. Lett. 70, 548 (1993); D. Vitali et al., Phys. Rev. Lett. 79, 2442 (1997); L. Viola et al., Phys. Rev. Lett. 82, 2417 (1999).

[6] S. Mancini et al., quant-ph/0108011.

[7] M. B. Plenio et al., Phys. Rev. A 59, 2468 (1999); A. Beige et al., J. Mod. Opt. 47, 2583 (2000); P. Horodecki, Phys. Rev. A 63, 022108 (2001).

[8] S. Bose et al., Phys. Rev. Lett. 83, 5158 (1999).

[9] A MATHEMATICA program is available from the authors that provides analytical expressions.

[10] L. Hardy, Contemp. Phys. 39, 419 (1998).

[11] G. Vidal and R.F. Werner, quant-ph/0102117; J. Eisert, Ph.D. thesis, Potsdam, 2001.

[12] C.H. Bennett et al., Phys. Rev. A 54, 3824 (1996); V. Vedral and M. B. Plenio, Phys. Rev. A 57, 1619 (1998).

[13] L. Gammaitoni et al., Rev. Mod. Phys. 70, 223 (1998).

[14] S. F. Huelga and M. B. Plenio, Phys. Rev. A 62, 052111 (2000); A. Buchleitner and R. N. Mantegna, Phys. Rev. Lett. 80, 3932 (1998).

[15] H. Nha et al., quant-ph/0106054.

[16] M. B. Plenio and P. L. Knight, Rev. Mod. Phys. 70, 101 (1998). 